Check for updates

Cite this: Phys. Chem. Chem. Phys., 2019, 21, 9433

Received 12th February 2019 Accepted 8th April 2019

DOI: $10.1039 / \mathrm{c} 9 \mathrm{cp} 00857 \mathrm{~h}$

rsc.li/pccp

\section{Prediction of the near-IR spectra of ices by ab initio molecular dynamics}

\author{
Rafael Escribano, (D) *a Pedro C. Gómez, ${ }^{b}$ Belén Maté, ${ }^{a}$ Germán Molpeceres ${ }^{a c}$ and \\ Emilio Artacho id ${ }^{d}$
}

\begin{abstract}
A method to predict the near-infrared spectra of amorphous solids by means of $a b$ initio molecular dynamics is presented. These solids can simulate molecular ices. To test the method, mixtures of methane, water and nitrogen are generated as amorphous samples of various concentrations. The full theoretical treatment includes as a first step, the optimization of their geometrical structure for a range of densities, after which, the most stable systems are taken as initial structures for molecular dynamics, performed at $200 \mathrm{~K}$ in trajectories of $4 \mathrm{ps}$ duration with a $0.2 \mathrm{fs}$ time step. All the dynamics are carried out using the first principles method, solving the quantum problem for the electrons using density-functional theory (DFT), and integrating the DFT forces, following the Born-Oppenheimer dynamics. After the dynamics, near-IR spectra are predicted by the Fourier transform of the macroscopic polarization autocorrelation function. The calculated spectra are compared with the experimental spectra of ice mixtures of $\mathrm{CH}_{4}$ and $\mathrm{H}_{2} \mathrm{O}$ recorded in our laboratory, and with some spectra recorded by the New Horizons mission on Pluto.
\end{abstract}

\section{Introduction}

The near-IR, covering wavelengths from 0.8 to $2.5 \mu \mathrm{m}$ approximately, corresponds to the absorption of overtones and combination bands of molecular vibrations. This is a particularly interesting IR region for many reasons, one of them being its extended use for the observation of astrophysical ices in the solar system. However, the theoretical simulation of spectra in this zone is a challenging task, especially for amorphous solids formed by a certain number of molecular units. High-level $a b$ initio methods that usually give reasonably accurate results for the description of anharmonic modes of individual molecules or small clusters are not easily applied to systems with several molecules, often linked among them by weak bonding of different types. We explore in this paper the application of $a b$ initio molecular dynamics (AIMD) calculations to predict the spectra of molecular ices in the near-IR region.

There have been some previous experimental investigations on solid mixtures containing $\mathrm{CH}_{4}, \mathrm{H}_{2} \mathrm{O}, \mathrm{N}_{2}$ and other volatiles in various proportions, ${ }^{1-7}$ even including the possibility of methane

\footnotetext{
${ }^{a}$ Instituto de Estructura de la Materia, IEM-CSIC, and Unidad Asociada Physical Chemistry UCM-CSIC, Serrano 123, 28006 Madrid, Spain.

E-mail: rafael.escribano@csic.es

${ }^{b}$ Departamento de Quimica Física, Facultad de C. Quimicas, Universidad Complutense, and Unidad Asociada Physical Chemistry UCM-CSIC, 28040 Madrid, Spain

${ }^{c}$ Institut für Theoretische Chemie, Universität Stuttgart, Pfaffenwaldring 55, D-70569, Stuttgart, Germany

${ }^{d}$ Theory of Condensed Matter, Cavendish Laboratory, University of Cambridge,

J.J. Thomson Ave., Cambridge, CB3 OHE, UK
}

clathrates, ${ }^{8}$ often with the aim of providing information for the analysis of astronomical data. The interest of these systems in the liquid phase goes beyond the astrophysical aspects, since e.g. solutions of $\mathrm{CH}_{4}$ in $\mathrm{H}_{2} \mathrm{O}$ are used in biological environments as a model of hydrophobic interactions relevant in many processes such as protein folding, ${ }^{9,10}$ and also in other branches of chemistry as in soft matter. Individual molecular components in this kind of ice formed by water plus non polar molecules are held together mainly by hydrogen bond interactions among the water molecules. They build up a sort of skeleton to which non polar components attach. Weak dispersive forces are responsible for the interactions among the non-polar molecules and among non-polar molecules with water molecules. The very nature of the interactions determines the methodological approach needed to describe the electron structure of the ices in the simulation of the spectra.

From the theoretical point of view, there is also abundant bibliography on the use of molecular dynamics to study molecules or clusters, and sometimes to calculate their vibrational spectra, ${ }^{11-15}$ but the specific application of this technique to predict overtone or combination bands, in the near-IR region, has seldom been discussed. ${ }^{13}$ AIMD calculations are not without difficulties, such as the lengthy explorations of the various parameters of the dynamics that must be selected and the choice of the electronic structure method employed to calculate interaction potentials. For the kind of sample that we plan to study here, where polar and non-polar molecules make part of the same structure, this is certainly an important issue.

Some of the most recent exciting results in astrophysics have come from the close observation of Pluto and other Trans- 
Neptunian Objects (TNO) by the NASA's New Horizons mission. Initial estimates of the surface composition of Pluto by Cruikshank et al. ${ }^{16}$ just before the arrival of the New Horizons spacecraft were followed by the publication of astonishingly remarkable spectra of several spots of Pluto's surface taken by the Ralph array of instruments. ${ }^{17}$ Later on, many of the results of this observation have been presented in several papers in a special issue of the Icarus journal. ${ }^{18}$ It has been found that ices of $\mathrm{CH}_{4}, \mathrm{H}_{2} \mathrm{O}, \mathrm{N}_{2}, \mathrm{CO}$ and possibly other species are present on Pluto at different concentrations with a widely diverse distribution. Ices formed by mixtures of these species and other molecules, like $\mathrm{NH}_{3}, \mathrm{CO}_{2}$ or $\mathrm{CH}_{3} \mathrm{OH}$, are also frequently found on other bodies of the solar system and in the interstellar medium. ${ }^{1,19-21}$

In this paper we continue previous theoretical studies ${ }^{3,22-25}$ on ices of astrophysical relevance, and intend now to theoretically simulate the near-IR spectra of these amorphous solid systems using molecular dynamics. A brief description of the most relevant methodological aspects is included in the next section. We have also recorded in our laboratory the spectra of ice mixtures of $\mathrm{CH}_{4}$ and $\mathrm{H}_{2} \mathrm{O}$, described in the following one. The Results section presents the application of the method to mixtures of $\mathrm{CH}_{4}, \mathrm{H}_{2} \mathrm{O}$ and $\mathrm{N}_{2}$ in different proportions. Finally, we compare the predicted spectra with those recorded in our lab and with some spectra from different spots on Pluto's surface.

\section{Methodology}

The use of molecular dynamics for the prediction of IR spectra has been discussed in a number of previous papers. ${ }^{11-15,26-28}$ and references therein, of which the one by Thomas et al. ${ }^{11}$ provides a very thorough discussion of the technique. In short, for individual molecules, the IR spectrum can be generated by the Fourier transform of the autocorrelation function of their dipole moment along the trajectory. In classical molecular dynamics simulations, the positions and velocities of the particles within a cell are described by means of force fields of diverse types. These are often based on additive contributions of several terms parameterized according to the interaction between the classically treated nuclei (harmonic terms for the bonds, Lennard-Jones for the interatomic potentials, etc.). Since we were mainly interested in dealing with weak spectral features arising from some 2nd order effects like overtones or combination bands, we chose ab initio molecular dynamics (AIMD) to try to use a better technique to follow the variation of interactions along the dynamics, without the constraint of parameterization. With AIMD, the electronic energies and interatomic forces calculated from the first-principles solution of the quantum problem posed by the electrons and the nuclei are displaced according to the classical trajectory defined by the first-principles forces.

In normal mode calculations of molecules, the IR absorption coefficient $S$ is proportional to the square of the derivatives of the dipole moment $\mu$ with respect to the normal coordinates $Q$ :

$$
S \propto\left(\frac{\partial \mu}{\partial Q}\right)^{2}
$$

In AIMD, the dipole moment derivatives are replaced by the dipole time-correlation function, and the Fourier transform is then carried out:

$$
A \propto \int\langle\dot{\mu}(\tau) \dot{\mu}(t+\tau)\rangle \mathrm{e}^{-\mathrm{i} \omega t} \mathrm{~d} t
$$

where $\dot{\mu}$ represents the time derivative of the dipole moment and $\omega$ is the frequency of the $Q$ normal mode, in $\mathrm{s}^{-1}$. Along the dynamics, all vibrational modes are excited simultaneously, and the information contained within the time-correlation function is extracted by its Fourier transform, with some similarity to the space distribution contained in an interferogram in Fourier Transform IR spectroscopy. For bulk solids, the dipole moment operator in eqn (1) and (2) needs to be replaced by the macroscopic polarization of the solid, ${ }^{29}$ which gives a suitable way to account for all charges within the unit cell.

Our models consisted of cubic cells where different numbers of $\mathrm{CH}_{4}, \mathrm{H}_{2} \mathrm{O}$ and $\mathrm{N}_{2}$ molecules were included generating threedimensional amorphous periodic structures in a Monte Carlo fashion, by minimizing close contacts between atoms, using the amorphous cell modulus of the Materials Studio package. ${ }^{30}$ Prior to the dynamics, we performed a geometry optimization of the structures inside the cell for a range of density values, looking for the structure and density which yielded the lowest optimization energy from a set of 10 amorphous structures. Consistency in the optimization was provided by checking that the frequencies of all fundamental vibrational modes were predicted as real numbers, indicating minima in their potential energy surface (PES). The selected structures at the minimum were taken for the dynamics process.

These initial optimizations were performed using the density functional theory (DFT) plane-wave pseudopotential method CASTEP $^{31}$ of the same Materials Studio package. ${ }^{30}$ We used for these initial structure relaxations the Perdew-Burke-Ernzerhof (PBE) functional with Grimme's D2 dispersion correction, ${ }^{32,33}$ with a plane wave basis set cut-off of $750 \mathrm{eV}$.

All the dynamics calculations were carried out using the Siesta implementation of DFT. ${ }^{34-36}$ Basis functions were taken from the literature. ${ }^{37}$ The Generalized Gradient Approximation (GGA) with revised Perdew-Burke-Ernzerhof (RPBE) ${ }^{38}$ functionals was chosen. Other parameters of the calculations were a mesh cutoff of 500 Ry giving a converged fine grid in real space for the real-space integrations needed to obtain the matrix elements of the Kohn-Sham Hamiltonian, ${ }^{35}$ and a $k$-grid cutoff of $6 \AA$, defining a sufficiently fine discretization of reciprocal space to approximate integrals over the Brillouin zone. ${ }^{39}$ The Siesta code was modified to calculate the macroscopic polarization of the samples at a specified number of steps in the dynamics. This was implemented to save computing time because the calculation of the polarization is a lengthy process. ${ }^{40}$ The polarization in a periodic solid up to a quantum of polarization, so that, in order for the results to be homogeneous, all values were referred to as the first quantum. The ensuing steps involved calculating the polarization auto-correlation function (PACF), 
normalizing the PACF and computing its Fourier transform that yields the IR spectrum.

The dynamics were established as a two-step procedure. First, the equilibration of the initial structure was achieved through an annealing process to a specified target temperature, starting from an initial configuration obtained as described above, and from random velocities corresponding to a Maxwell distribution for the target temperature, and letting it equilibrate following the dynamics of the system under a Berendsen thermostat. ${ }^{41}$ The structure at that temperature was selected from the output of geometries and was then taken as the starting point for the full dynamics process.

We chose a constant-volume/constant-energy/constantcomposition $(N V E)$ microcanonical ensemble integrated using a time discretization and the Verlet integration algorithm. ${ }^{41}$ This implies that our simulation cell was allowed to vary its temperature within reasonable margins, while keeping the cell size and internal energy constant, which allowed a closer reference to the conditions in some astronomical media. The equilibration temperature was $200 \mathrm{~K}$, higher than the estimated value of many astronomical samples, but necessary to excite adequately the very weak vibrational motions that appear in the near-IR region. In practice, we are primarily interested in the relative intensities of spectral features arising from the different components of the sample, mainly methane and water, and not in their absolute intensity, which would require a completely different treatment of the problem, and therefore the choice of $200 \mathrm{~K}$ seems justified.

Choosing the time step for the dynamics is a fundamental issue. It's in an inverse ratio to the frequency of the modes that one wants to sample. For the fundamental $\mathrm{O}-\mathrm{H}$ and $\mathrm{C}-\mathrm{H}$ stretching modes, which appear at $\sim 3000 \mathrm{~cm}^{-1}$, or $\sim 100 \mathrm{THz}$, time steps of $1 \mathrm{fs}$ or $0.5 \mathrm{fs}$ are usually chosen. Since we were interested in higher frequency modes, we had to reduce the step, but this also increased considerably the computer running time. After checking for $0.2 \mathrm{fs}$ and $0.1 \mathrm{fs}$, we decided that the gain for the shortest step was not large enough to justify the much longer computing time, so we selected $0.2 \mathrm{fs}$. In a similar manner, the length of the dynamics fixes the resolution of the spectra. As the resolution of the observed spectra of many astronomical samples is not very large, compared with laboratory measurements, we tried lengths of 2 ps and 4 ps for our trajectories, and finally opted for the second choice for most of our runs, which therefore means dynamics of 20000 steps. In some particular cases, longer dynamics were performed looking for some increased resolution. For illustration, and to show the consistent behavior of the calculations, we include in Fig. 1 an example of the resolution gain between dynamics of 2 ps and 6.3 ps on the spectra of a sample with $5 \mathrm{CH}_{4}$ and $1 \mathrm{H}_{2} \mathrm{O}$ molecules. The predicted spectra achieve a higher degree of resolution, as can be seen in Fig. 1, where several bands are resolved at each stage. Between 2 ps and 4 ps, the most obvious case is the splitting of the main feature, at $\sim 6300 \mathrm{~cm}^{-1}$, into two components, at $6100 \mathrm{~cm}^{-1}$ and $6600 \mathrm{~cm}^{-1}$, with other splittings for the bands at $5400 \mathrm{~cm}^{-1}$ and $4100 \mathrm{~cm}^{-1}$. Between 4 ps and 6.3 ps, many individual bands are disclosed in the whole region. The assignments of all these bands will be

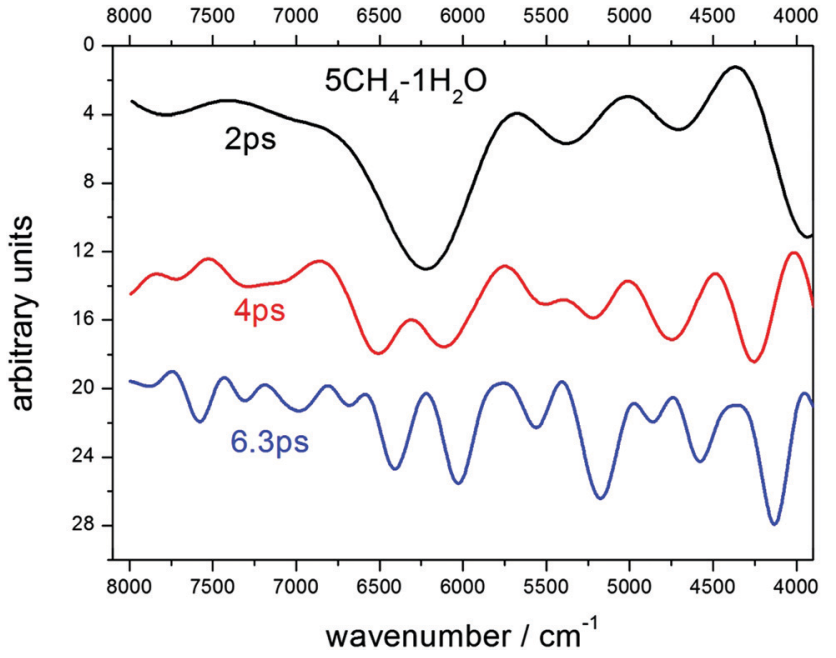

Fig. 1 Spectra predicted after 3 different dynamics of a sample with $5 \mathrm{CH}_{4}-1 \mathrm{H}_{2} \mathrm{O}$ molecules. An increase in the length of the run from $2 \mathrm{ps}$ through 6.3 ps allows resolving most of the individual bands. Spectra are offset along the ordinate axis for clarity.

discussed below. However, the 6.3 ps dynamics conveys a very lengthy computation, so we finally accepted 4 ps as the most adequate length for our purposes.

A few considerations must be borne in mind regarding the spectra calculated via molecular dynamics for this near-IR region. First, it is important to stress that the vibrations in this IR window are intrinsically non-linear, inasmuch as they correspond to excitations of two or more normal modes of the same (overtones) or different vibrations (combination bands). The simulation of non-linearity is achieved through the autocorrelation process of the macroscopic polarization that is obtained in a linear calculation. Next, the wavenumber accuracy expected from these calculations cannot be as high as that which can be reached by high level ab initio methods for the fundamental modes of single molecules. To begin with, the basis sets and theoretical parameters used for solving the electronic structure at each stage in our dynamics are chosen to achieve a fast convergence, a constraint that can be waived for non-dynamics ab initio calculations, like those often applied to deal with simple molecules. Moreover, the anharmonicity of the vibrational modes implies wavenumber shifts from the harmonic prediction which are not easy to reproduce. Another important aspect of the spectra concerns the width of the observed lines. All experimental $\mathrm{H}_{2} \mathrm{O}$ bands are usually very broad, often spanning more than $200 \mathrm{~cm}^{-1}$, and this is not reproduced in the calculations, where broadening effects induced by H-bonding, for instance, are not specifically taken into account. Finally, the consideration of an amorphous solid as a repetitive unit of a single cell implies in itself some simplifications over the real structure. Taking all this into account, wavenumber predictions with an accuracy of $\pm 100 \mathrm{~cm}^{-1}$ may be considered satisfactory, again for this IR region and this type of vibration.

From the calculation point of view, the inclusion of more molecules within the simulation cell, keeping the $\mathrm{CH}_{4} / \mathrm{H}_{2} \mathrm{O}$ ratio, could change the aspect of some vibrations. An example 


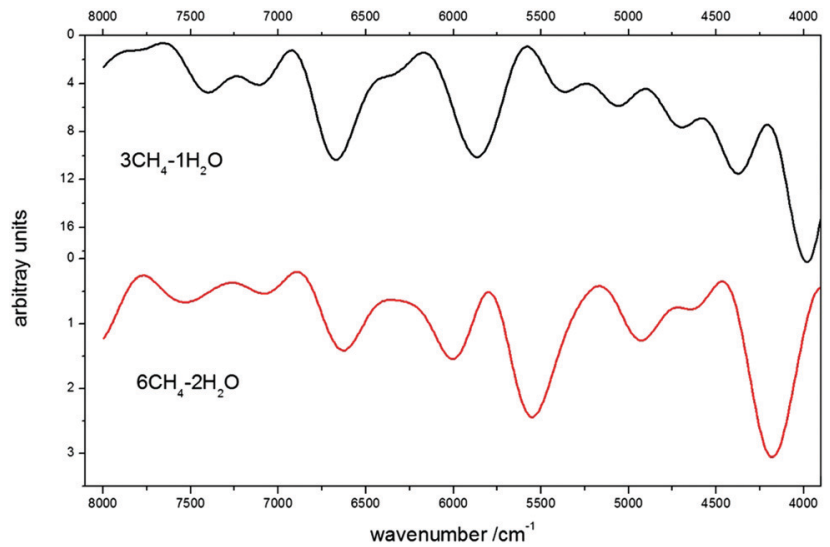

Fig. 2 Spectra of two samples with the same ratio of $\mathrm{CH}_{4} / \mathrm{H}_{2} \mathrm{O}$ molecules. Some differences can be attributed to shifts in $\mathrm{H}_{2} \mathrm{O}$ vibrations involving $\mathrm{H}$-bonding and to broadening of $\mathrm{CH}_{4}$ bands.

is given in Fig. 2, where a comparison is presented for two dynamics with a $\mathrm{CH}_{4} / \mathrm{H}_{2} \mathrm{O}$ ratio of $3 / 1$, namely $3 \mathrm{CH}_{4}-1 \mathrm{H}_{2} \mathrm{O}$ and $6 \mathrm{CH}_{4}-2 \mathrm{H}_{2} \mathrm{O}$ samples. In the second case, with 2 water molecules, some wavenumber displacements could be expected, especially for the $\mathrm{O}-\mathrm{H}$ vibrations where $\mathrm{H}$-bond effects may be important. On the other hand, the $\mathrm{CH}_{4}$ bands should not shift in the spectrum, but the presence of more molecules in the simulation cell may blur the contour, yielding an apparent loss of resolution. For those samples where very little water is expected, it seems safe to work with models with only one $\mathrm{H}_{2} \mathrm{O}$ molecule and a growing number of $\mathrm{CH}_{4}$ molecules, as discussed below. On the other hand, for the comparison with water-rich samples, models with more $\mathrm{H}_{2} \mathrm{O}$ molecules should be used.

\section{Experimental}

Our laboratory is equipped with a high vacuum chamber with a closed-cycle He cryostat, coupled to a Bruker Vertex70 FTIR spectrometer. The setup has been described in detail in a previous article. ${ }^{42}$ Methane and water ice mixtures are grown by vapor deposition on an infrared transparent Si substrate cooled to $30 \mathrm{~K}$. The gases are introduced into the chamber through independent lines, one provided with a Alicat mass flow controller and the other with a needle valve, used for $\mathrm{CH}_{4}$ and $\mathrm{H}_{2} \mathrm{O}$, respectively. The pressure in the chamber is $\sim 10^{-8}$ mbar before deposition and increases to $10^{-5}-10^{-4} \mathrm{mbar}$ during the deposits. We grow ice layers between 6 and $15 \mu \mathrm{m}$ thick at rates that vary from 2 to $5 \mu \mathrm{m} \mathrm{h}^{-1}$. In order to control the $\mathrm{CH}_{4} / \mathrm{H}_{2} \mathrm{O}$ ratio in the mixtures we have calibrated the pressure of each gas in the chamber in the following way. First, we record the spectra of the pure species in the mid-IR and estimate the number of molecules in the sample by reference to literature band strengths of the main bands: $A=1.9 \times 10^{-16} \mathrm{~cm}$ per molecule for the $\mathrm{H}_{2} \mathrm{O}$ band at $3200 \mathrm{~cm}^{-1}, 4$ and $A=8.4 \times 10^{-18} \mathrm{~cm}$ per molecule for the $\mathrm{CH}_{4}$ band at $1300 \mathrm{~cm}^{-1} \cdot{ }^{43}$ With these values we can estimate the growing rate of the corresponding ices, and from the growing rates, assuming a sticking coefficient of 1 in

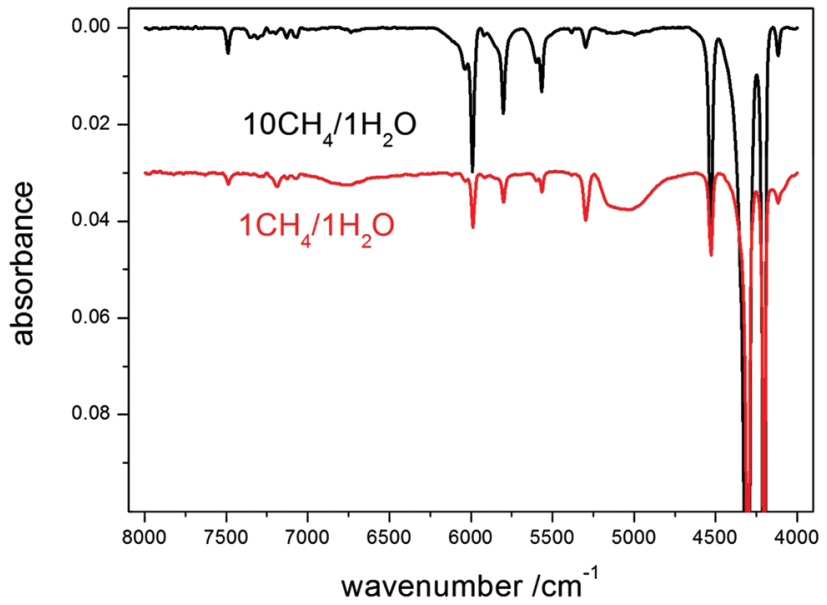

Fig. 3 Laboratory spectra of mixtures of $\mathrm{CH}_{4}$ and $\mathrm{H}_{2} \mathrm{O}$ in ratios of 10/1 (black, above) and 1/1 (red, offset). The absorbance axis has been cut off for a better view of the weaker bands at higher wavenumbers.

agreement with previous literature, ${ }^{44}$ we calculate the gas pressure. In our experiments, we have recorded spectra in the near-IR region using a near-IR lamp with a MCT detector, at $8 \mathrm{~cm}^{-1}$ resolution, adding 300 scans, and covering a spectral range from 8000 to $2000 \mathrm{~cm}^{-1}$.

We have recorded the spectra of samples of pure $\mathrm{CH}_{4}$, co-deposited $\mathrm{CH}_{4} / \mathrm{H}_{2} \mathrm{O}$ mixtures in ratios of $10 / 1$ and $1 / 1$, and pure $\mathrm{H}_{2} \mathrm{O}$ ice, at $30 \mathrm{~K}$. The spectra of the pure species are used for comparison and assignments; we present in Fig. 3 the spectra of the mixtures in the region of maximum interest. A baseline with the contour of IR interference fringes has been subtracted from the recorded trace. The $\mathrm{CH}_{4}$ features are sharp and stand out for both mixtures, whereas the $\mathrm{H}_{2} \mathrm{O}$ bands are broad and it is not easy to assert their band center. From the higher water-content sample, we estimate two $\mathrm{H}_{2} \mathrm{O}$ band centers at $\sim 5100$ and $6760 \mathrm{~cm}^{-1}$, with a further less obvious feature at $6400 \mathrm{~cm}^{-1}$. Later on in this paper we collect in a table the assignment of the main bands observed in our experiments, together with values from the calculated spectra.

\section{Results}

We prepared models with a large variation of $\mathrm{CH}_{4}$ and $\mathrm{H}_{2} \mathrm{O}$ ratios, from the individual species themselves to mixtures with different $\mathrm{CH}_{4} / \mathrm{H}_{2} \mathrm{O}$ content, plus more samples with $\mathrm{N}_{2}$ added, to investigate its possible effect on the spectra. We had in mind the possible application of this work to study spectra in astronomical environments. Thus, we chose samples using three strategies: first, with growing $\mathrm{CH}_{4}$ content, for $\mathrm{CH}_{4} / \mathrm{H}_{2} \mathrm{O}$ values from $3 / 1$ to $10 / 1$; then, with growing $\mathrm{H}_{2} \mathrm{O}$ content, for values 10/2 (which allowed also direct comparison with the 10/1 case), 3/3, 3/6 and 3/9; and finally, for $\mathrm{N}_{2}$-containing samples of various compositions. Only spectra that have direct usefulness in the following discussion will be reproduced in the figures of this paper.

For each model, we performed optimization of the geometry for several density values, in the range between 0.45 and $1.0 \mathrm{~g} \mathrm{~cm}^{-3}$. 
Table 1 Summary of the results of the most stable samples generated for the molecular mixtures studied in this work. The molecules were contained in cubic cells of side a (2nd column), for which the corresponding density is $\rho$ (3rd column). The listed structures are those that yielded a minimum optimization energy $E$ (4th column). The last column lists the formation energy calculated using eqn (3)

\begin{tabular}{llllr}
\hline Molecular system & $a / \AA$ & $\rho / \mathrm{g} \mathrm{cm}^{-3}$ & $E / \mathrm{eV}$ & $\Delta E / \mathrm{eV}$ \\
\hline $3 \mathrm{CH}_{4}-1 \mathrm{H}_{2} \mathrm{O}$ & 5.53 & 0.65 & -1122.615 & -0.587 \\
$5 \mathrm{CH}_{4}-1 \mathrm{H}_{2} \mathrm{O}$ & 6.308 & 0.65 & -1561.997 & -0.742 \\
$6 \mathrm{CH}_{4}-2 \mathrm{H}_{2} \mathrm{O}$ & 6.965 & 0.65 & -2245.263 & -1.207 \\
$10 \mathrm{CH}_{4}-1 \mathrm{H}_{2} \mathrm{O}$ & 7.696 & 0.65 & -2660.431 & -1.108 \\
$10 \mathrm{CH}_{4}-2 \mathrm{H}_{2} \mathrm{O}$ & 7.947 & 0.65 & -3124.476 & -1.966 \\
$3 \mathrm{CH}_{4}-3 \mathrm{H}_{2} \mathrm{O}$ & 6.391 & 0.65 & -2049.833 & -1.431 \\
$3 \mathrm{CH}_{4}-6 \mathrm{H}_{2} \mathrm{O}$ & 7.363 & 0.65 & -3441.204 & -3.241 \\
$3 \mathrm{CH}_{4}-9 \mathrm{H}_{2} \mathrm{O}$ & 8.129 & 0.65 & -4832.555 & -5.030 \\
$5 \mathrm{CH}_{4}-1 \mathrm{H}_{2} \mathrm{O}-3 \mathrm{~N}_{2}$ & 6.954 & 0.90 & -3175.189 & 2.948 \\
$3 \mathrm{CH}_{4}-3 \mathrm{H}_{2} \mathrm{O}-3 \mathrm{~N}_{2}$ & 7.004 & 0.90 & -3667.327 & -2.043 \\
$3 \mathrm{CH}_{4}-3 \mathrm{H}_{2} \mathrm{O}-6 \mathrm{~N}_{2}$ & 7.656 & 1.0 & -5284.375 & -2.209 \\
$3 \mathrm{CH}_{4}-6 \mathrm{H}_{2} \mathrm{O}-6 \mathrm{~N}_{2}$ & 8.136 & 1.0 & -6675.189 & -3.462 \\
& & & &
\end{tabular}

Table 1 collects the results of the lowest-energy structures for some of our samples. It is these structures that were taken as initial values for the dynamics process. We also list in Table 1 the formation energy of the models, calculated as:

$$
\Delta E=E(\text { mol.sys. })-m \times E\left(\mathrm{CH}_{4}\right)-n \times E\left(\mathrm{H}_{2} \mathrm{O}\right)-p \times E\left(\mathrm{~N}_{2}\right)
$$

where $E$ (mol.sys.) is the energy of the fully optimized structure, $m \mathrm{CH}_{4}-n \mathrm{H}_{2} \mathrm{O}-p \mathrm{~N}_{2}$, listed in the 4 th column of Table 1 , and $E\left(\mathrm{CH}_{4}\right), E\left(\mathrm{H}_{2} \mathrm{O}\right)$ and $E\left(\mathrm{~N}_{2}\right)$ are those of individual $\mathrm{CH}_{4}, \mathrm{H}_{2} \mathrm{O}$ and $\mathrm{N}_{2}$ molecules, respectively, all of them optimized at the same level of theory as the full model. The most interesting result is the largest stabilization achieved when a second $\mathrm{H}_{2} \mathrm{O}$ molecule is added to the $10 \mathrm{CH}_{4}-1 \mathrm{H}_{2} \mathrm{O}$ system, and also when $3 \mathrm{H}_{2} \mathrm{O}$ molecules are added to the $3 \mathrm{CH}_{4}-3 \mathrm{H}_{2} \mathrm{O}-6 \mathrm{~N}_{2}$ sample, probably due to the generation of $\mathrm{H}$-bonds among the $\mathrm{H}_{2} \mathrm{O}$ molecules.

The analysis of Table 1 allows us to draw some further conclusions. In the first place, it can be seen that the variation of the formation energy of the methane-water mixtures is not exactly linear with an increase in one molecule of either of them. However, the addition of $\mathrm{H}_{2} \mathrm{O}$ contributes an amount of energy in the range of 0.4 to $0.8 \mathrm{eV}$ per molecule, which is notably larger than the $0.07-0.08 \mathrm{eV}$ value per molecule for $\mathrm{CH}_{4}$. For the mixtures containing $\mathrm{N}_{2}$ in addition to $\mathrm{H}_{2} \mathrm{O}$ and $\mathrm{CH}_{4}$, the individual contribution of $\mathrm{N}_{2}$ to the energy formation is somewhat more difficult to assess from the data in the table, but it may be close to $0.06 \mathrm{eV}$ per molecule. All this is consistent with the picture of the clusters being formed by a backbone of water molecules linked by H-bond interactions to which the non-polar species are attached.

For the sake of the forthcoming discussion, we reproduce in Fig. 4 the calculated spectrum of the $3 \mathrm{CH}_{4}-3 \mathrm{H}_{2} \mathrm{O}$ sample. This spectrum is chosen because the most important modes of each species can be seen, which facilitates the comparison with the'spectra of samples with higher or lower abundance of either molecule. To summarize this part, Table 2 collects a survey of the main $\mathrm{CH}_{4}$ and $\mathrm{H}_{2} \mathrm{O}$ vibrations in the $4000-8000 \mathrm{~cm}^{-1}$ region, with experimental wavenumbers from the observed spectra in our laboratory together with the calculated wavenumbers

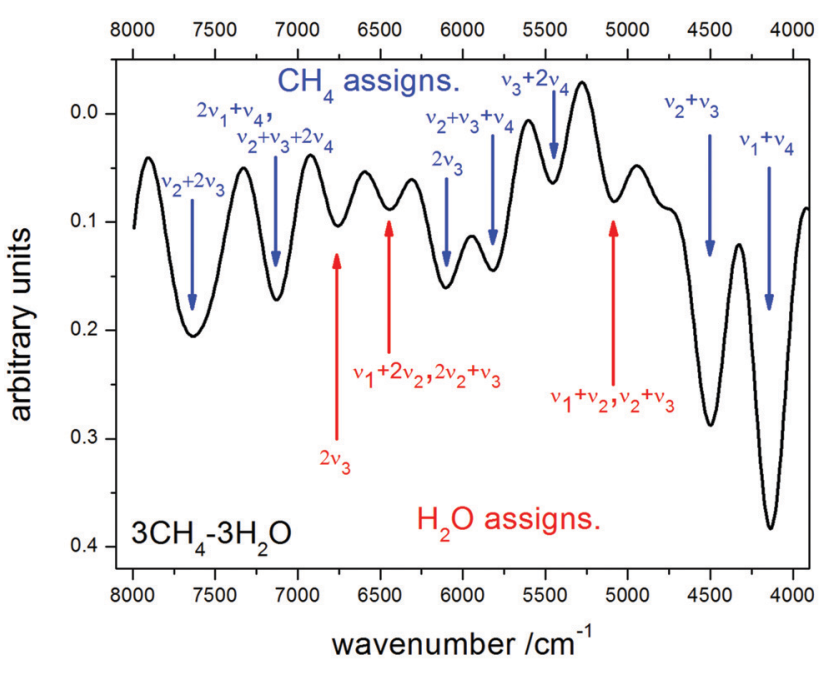

Fig. 4 Calculated spectrum of a sample with $3 \mathrm{CH}_{4}-3 \mathrm{H}_{2} \mathrm{O}$ molecules, taken as the basis for the assignments listed in Table 2 and for the discussion on the relative intensities of $\mathrm{CH}_{4}$ and $\mathrm{H}_{2} \mathrm{O}$ spectral features.

Table 2 Main features in the near-IR region of the spectra of $\mathrm{CH}_{4}$ and $\mathrm{H}_{2} \mathrm{O}$ mixtures and tentative assignments. Successive columns display wavenumber values measured from the spectra recorded in our laboratory; tentative assignments for $\mathrm{CH}_{4}$ and $\mathrm{H}_{2} \mathrm{O}$ bands from the indicated references, and wavenumbers calculated in this work, taken from the $3 \mathrm{CH}_{4}-3 \mathrm{H}_{2} \mathrm{O}$ sample (see Fig. 4)

\begin{tabular}{|c|c|c|c|}
\hline $\begin{array}{l}\text { Lab. observed } \\
\text { (this work) } / \mathrm{cm}^{-1}\end{array}$ & $\begin{array}{l}\text { Assignment } \\
\mathrm{CH}_{4}{ }^{a}\end{array}$ & $\begin{array}{l}\text { Assignment } \\
\mathrm{H}_{2} \mathrm{O}^{b}\end{array}$ & $\begin{array}{l}\text { Calculated } \\
\text { (this work) } / \mathrm{cm}^{-1}\end{array}$ \\
\hline 4116 & $\nu_{2}+2 \nu_{4}$ & & \\
\hline 4206 & $\nu_{1}+\nu_{4}$ & & 4144 \\
\hline 4303 & $\nu_{3}+\nu_{4}$ & & \\
\hline 4530 & $\nu_{2}+\nu_{3}$ & & 4503 \\
\hline 5100 & & $\begin{array}{l}\nu_{1}+\nu_{2} \\
\nu_{2}+\nu_{3}\end{array}$ & 5088 \\
\hline 5383 & $\nu_{2}+3 \nu_{4}$ & & \\
\hline 5564 & $\nu_{3}+2 \nu_{4}$ & & 5448 \\
\hline 5596 & $2 \nu_{2}+2 \nu_{4}$ & & \\
\hline 5801 & $\nu_{2}+\nu_{3}+\nu_{4}$ & & 5818 \\
\hline 5930 & $2 \nu_{1}^{c}$ & & \\
\hline 5993 & $2 \nu_{3}$ & & 6099 \\
\hline 6040 & $2 \nu_{2}+\nu_{3}$ & & \\
\hline $6400 \mathrm{br}^{d}$ & & $\begin{array}{l}\nu_{1}+2 \nu_{2} \\
\nu_{3}+2 \nu_{2}\end{array}$ & 6448 \\
\hline $6760 \mathrm{br}$ & & $2 \nu_{3}$ & 6763 \\
\hline $\begin{array}{l}7065 \\
7124^{e}\end{array}$ & $2 \nu_{1}+\nu_{4} ; \nu_{2}+\nu_{3}+2 \nu_{4}$ & & 7133 \\
\hline 7180 & $\nu_{1}+\nu_{2}+\nu_{3}$ & & \\
\hline 7484 & $\nu_{2}+2 \nu_{3}$ & & 7639 \\
\hline
\end{tabular}

${ }^{a}$ Ref. $5-7 .{ }^{b}$ Ref. 5 and $48 .{ }^{c}$ Tentative assignment. This mode is IRforbidden, but could be activated in $\mathrm{CH}_{4}-\mathrm{H}_{2} \mathrm{O}$ mixtures. ${ }^{d} \mathrm{Br}$ indicates the approximate band origin in broad features. ${ }^{e}$ Unassigned.

reported in this work, taken from the peaks shown in Fig. 4. The quoted assignments are based on previous works (see caption to Table 2). We have indicated with blue arrows in Fig. 4 the peaks assigned to $\mathrm{CH}_{4}$ and with red arrows those assigned to $\mathrm{H}_{2} \mathrm{O}$.

\section{Application to the spectra from Pluto}

The near-IR spectra recorded by the New Horizons instruments provide a good opportunity to test the validity or usefulness 
of our method. These spectra have been analyzed in great detail by the members of the New Horizons Science Team ${ }^{45,46}$ by means of Principal Component Analysis and the transfer model of Hapke, ${ }^{47}$ in terms of various parameters like the relative concentration of $\mathrm{N}_{2}, \mathrm{CH}_{4}, \mathrm{H}_{2} \mathrm{O}$ and $\mathrm{CO}$ ices, tholins, grain size and visible dark red material, revealing their complex structure. We therefore do not intend to accurately reproduce them here, but to check how closely we can predict the relative strength of $\mathrm{CH}_{4}$ and $\mathrm{H}_{2} \mathrm{O}$ bands by using our simple model containing various proportions of these molecules, and also to investigate the spectral effects produced by the presence of $\mathrm{N}_{2}$. In particular, we have focused on two of the spectra presented in Fig. 3 of Grundy et al., ${ }^{17}$ traces a and d, corresponding to Plutos's North Pole and to the area around the Pulfrich crater, respectively.

Fig. 5 displays the spectra of three mixtures with growing $\mathrm{CH}_{4}$ content, for $\mathrm{CH}_{4} / \mathrm{H}_{2} \mathrm{O}$ ratios of $3 / 1,5 / 1$ and $10 / 1$. We also include the outline of the spectrum of trace a in Fig. 3 of Grundy et al., ${ }^{17}$ scanned from the original figure and transformed to the linear scale in wavenumber for comparison. None of the calculated spectra matches perfectly the observation, but we can draw some conclusions from a piecewise analysis of the figure. The contour of the experimental spectrum in the lower wavenumber region, between 4000 and $5000 \mathrm{~cm}^{-1}$, is better reproduced in the spectrum of the intermediate sample, with $5 \mathrm{CH}_{4}-1 \mathrm{H}_{2} \mathrm{O}$. However, in the $5500-6000 \mathrm{~cm}^{-1}$ and $7000-7500 \mathrm{~cm}^{-1}$ zones, the spectrum of the $10 \mathrm{CH}_{4}-1 \mathrm{H}_{2} \mathrm{O}$ sample seems to have a closer resemblance to the observed trace than that of the other samples. The match would be even closer if we impose a shift to a higher wavenumber of the order of $100 \mathrm{~cm}^{-1}$ on the calculated results. Such a shift may be acceptable for theoretical calculations of this level of accuracy, involving overtones and combination bands (see Table 2) as indicated above. With respect to the contribution of water bands, it is almost negligible in the experimental spectrum,

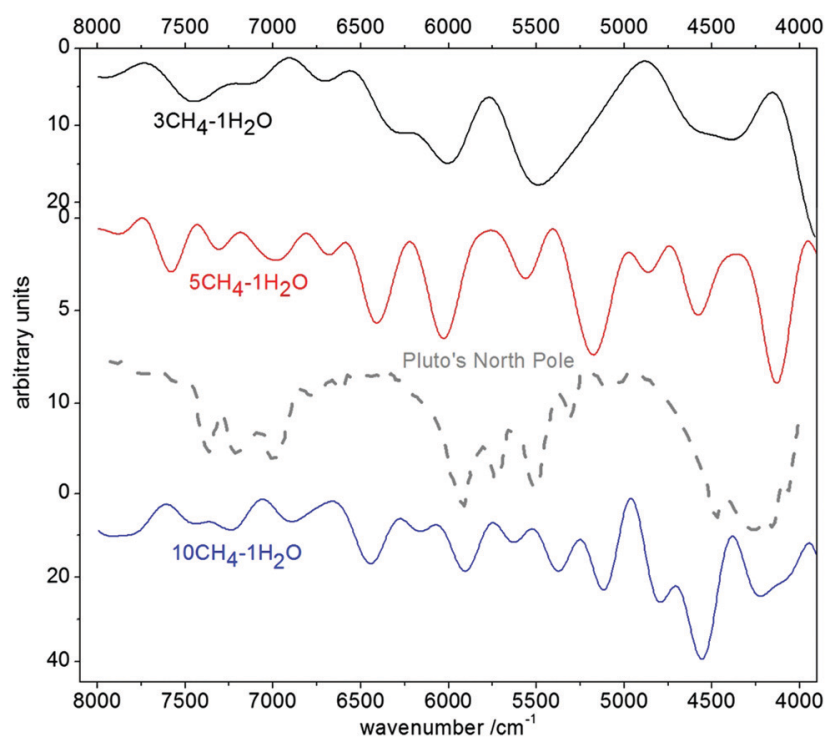

Fig. 5 Calculated spectra of three samples with increasing $\mathrm{CH}_{4}$ proportion, together with the adapted trace a of Fig. 3 of Grundy et al. ${ }^{17}$ in contrast to the appearance of strong water features in the two water-rich theoretical samples, top two traces in Fig. 5. Such features are weaker in the bottom trace, although they may still be fairly large for the comparison with the experiment. In conclusion, we estimate that the $\mathrm{CH}_{4}$ to $\mathrm{H}_{2} \mathrm{O}$ ratio in the North Pole of Pluto is probably around 10/1, but values in the $8 / 1$ or $12 / 1$ vicinity cannot be discarded.

Going in the opposite direction, we studied the effect of growing $\mathrm{H}_{2} \mathrm{O}$ content in the mixture, using as a reference the spectrum of the Pulfrich crater in Pluto, trace $d$ in Fig. 3 of Grundy et al. $^{17}$ We present in Fig. 6 the spectra of three samples, with $3 \mathrm{CH}_{4}-3 \mathrm{H}_{2} \mathrm{O}, 3 \mathrm{CH}_{4}-6 \mathrm{H}_{2} \mathrm{O}$ and $3 \mathrm{CH}_{4}-9 \mathrm{H}_{2} \mathrm{O}$ molecules. Comparison of the $3 / 3$ and $3 / 6$ spectra (top two traces) is fairly straightforward, with an increase in the intensity of the main $\mathrm{H}_{2} \mathrm{O}$ bands, at $\sim 5100,6400$ and $6740 \mathrm{~cm}^{-1}$, with respect to $\mathrm{CH}_{4}$ neighboring features. However, interestingly, comparison of the $3 / 6$ and $3 / 9$ spectra is not so obvious. The $\mathrm{H}_{2} \mathrm{O}$ bands at the higher wavenumber side grow both in intensity and width, overlapping with nearby $\mathrm{CH}_{4}$ features with the net effect that the 3/9 spectrum appears as a less resolved trace. The observed spectrum at the Pulfrich crater of Pluto shows very broad $\mathrm{H}_{2} \mathrm{O}$ bands with a smaller structure of narrow lines of $\mathrm{CH}_{4}$ in the 5500-6000 and $7000-7500 \mathrm{~cm}^{-1}$ regions. Keeping in mind the inability of the current method to correctly reproduce the wide $\mathrm{H}_{2} \mathrm{O}$ bands observed experimentally, we estimate that the $\mathrm{CH}_{4} / \mathrm{H}_{2} \mathrm{O}$ mixing ratio that better resembles that of the Pulfrich crater would be between $1 / 2$ and $1 / 3$.

It is worth noting that our estimates for the ratio of $\mathrm{CH}_{4}$ and $\mathrm{H}_{2} \mathrm{O}$ in the cases studied here match reasonably well the results quoted in Table 3 of Protopapa et $a .^{46}$ from their analysis, where their models a and e correspond to the spectra of the Pulfrich crater and North Pole of Pluto, respectively.

Finally, we investigated the effect of adding $\mathrm{N}_{2}$ molecules on the spectra. Nitrogen is assumed to be abundant in many

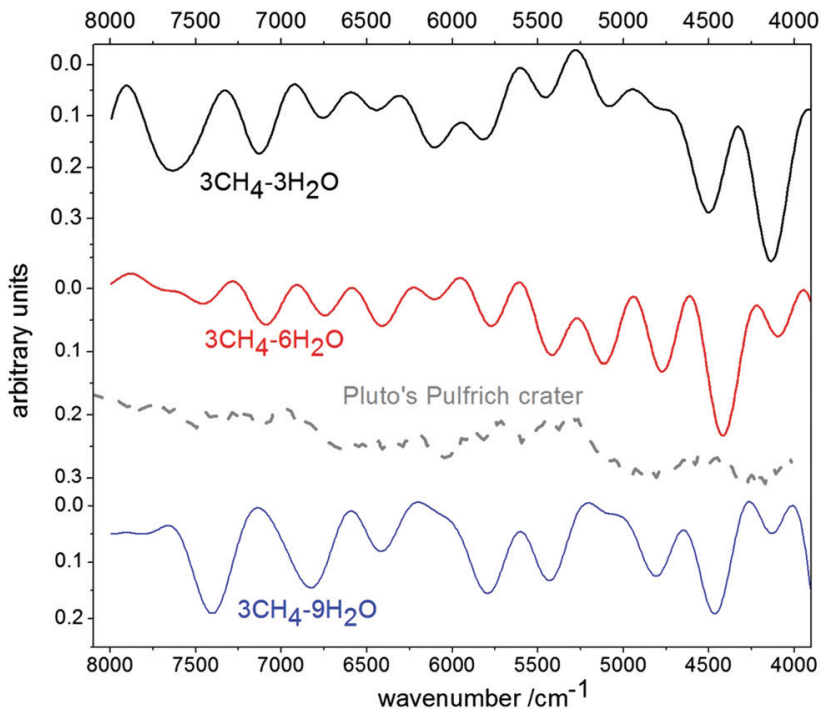

Fig. 6 Calculated spectra of mixtures with increasing $\mathrm{H}_{2} \mathrm{O}$ proportion, in comparison with the spectrum at the Pulfrich crater of Pluto, adapted from trace d of Fig. 3 of Grundy et al. ${ }^{17}$ 


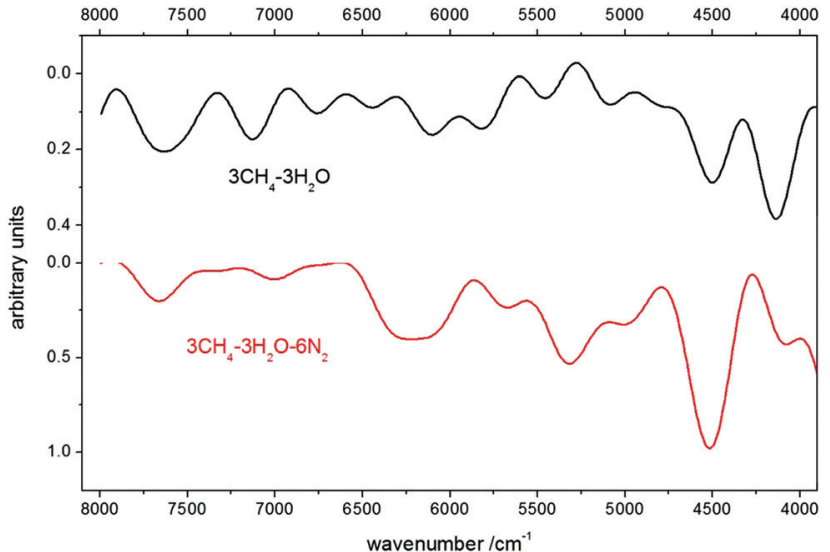

Fig. 7 Comparison of the calculated spectra of two samples with the same $\mathrm{CH}_{4} / \mathrm{H}_{2} \mathrm{O}$ content and with and without $\mathrm{N}_{2}$, namely $3 \mathrm{CH}_{4}-3 \mathrm{H}_{2} \mathrm{O}$ and $3 \mathrm{CH}_{4}-3 \mathrm{H}_{2} \mathrm{O}-6 \mathrm{~N}_{2}$. The main effect of introducing $\mathrm{N}_{2}$ molecules in the sample is to blur the total spectrum of the mixture.

regions of Pluto, forming $\mathrm{N}_{2}$-rich/ $\mathrm{CH}_{4}$-rich ices of varying proportions, even giving rise to a weak peak observed in some of the traces of Fig. 3 of Grundy et al. ${ }^{17}$ at $\sim 4650 \mathrm{~cm}^{-1}$, corresponding to the first harmonic of its IR-forbidden fundamental vibration, at $\sim 2330 \mathrm{~cm}^{-1}$. To check its possible effect on the calculated spectra, we designed further samples based on some of the models described above, with the inclusion of $3 N_{2}$ or $6 N_{2}$ molecules, which induced a corresponding density increase. The subsequent dynamics yielded the predicted spectra. As an example, we collect in Fig. 7 the spectra of pairs $3 \mathrm{CH}_{4}-3 \mathrm{H}_{2} \mathrm{O}$ and $3 \mathrm{CH}_{4}-3 \mathrm{H}_{2} \mathrm{O}-6 \mathrm{~N}_{2}$, to discuss the observed effects. The presence of $\mathrm{N}_{2}$ molecules induces a general blurring of the spectral features, responsible for the apparent intensity gain of the $4500 \mathrm{~cm}^{-1}$ band. This cannot in any case be attributed to the very weak peak experimentally measured at $\sim 4650 \mathrm{~cm}^{-1}$, as mentioned above, which we could not expect to observe in our simulation, where strong $\mathrm{CH}_{4}$ and $\mathrm{H}_{2} \mathrm{O}$ bands dominate. The blurring could be foreseen as a consequence of the presence of a relatively large number of non-polar species in the simulation cell encountered by the methane or water molecules during their motion, inducing a large inhomogeneity in their environment.

\section{Conclusions}

We describe in this paper the use of $a b$ initio molecular dynamics as a tool to predict spectra in the near-IR region for molecular solids, with possible application to astronomical ices. The high frequency of this zone compared to mid-IR requires the use of a short time step for the dynamics. On the other hand, the length of the trajectory can be adjusted to achieve a balance between the expected resolution, directly related to the duration of the dynamics, and the computer resources available. For our calculations, we have found that a time step of 0.2 fs over a 4 ps trajectory was a reasonable choice. Although the surface temperature of many astrophysical objects is quite low, we chose to perform our dynamics at an initial temperature of $200 \mathrm{~K}$, because bands in the near-IR region are generally weak and the weaker features would be almost negligible at lower temperatures. All dynamics are performed using a modification of the Siesta code. The Fourier transform of the autocorrelation function of the macroscopic polarization calculated along the trajectory yields the vibrational spectrum.

We have applied this method to the study of ice mixtures of $\mathrm{CH}_{4}, \mathrm{H}_{2} \mathrm{O}$ and $\mathrm{N}_{2}$, species frequently present on astronomical samples. We have built theoretical models of amorphous solids covering a broad range of $\mathrm{CH}_{4}$ to $\mathrm{H}_{2} \mathrm{O}$ ratios, from 10/1 to 3/9. The models consist of simulation cells, where the molecules are introduced, and prior to the dynamics, they are relaxed for a range of density values, looking for a minimum in their PES. Analysis of the formation energies shows an increase in the stabilization of the sample when more than one $\mathrm{H}_{2} \mathrm{O}$ molecule is present, probably due to the formation of $\mathrm{H}$-bonds among them. Then, the most stable structures are annealed at $200 \mathrm{~K}$ to yield an equilibrated structure, which is subsequently subjected to the dynamics trajectory. From the results of the dynamics, the spectra of our samples in the near-IR region are predicted.

To complement our calculations, we have recorded in our lab the spectra of ices of pure $\mathrm{CH}_{4}$ and $\mathrm{H}_{2} \mathrm{O}$, and of ice mixtures with ratios of $10 / 1$ and $1 / 1$. We have compared the predictions of our method with our experimental spectra, and with spectra recorded by the New Horizons NASA mission. In particular, for two spots of Pluto's surface which show the largest variation in relative $\mathrm{CH}_{4} / \mathrm{H}_{2} \mathrm{O}$ content, we estimate approximate $\mathrm{CH}_{4} / \mathrm{H}_{2} \mathrm{O}$ ratios in reasonable agreement with the published results.

Possible extensions of this work may include the addition of more molecules in our samples and the study of mixed amorphous/crystalline species.

\section{Conflicts of interest}

There are no conflicts to declare.

\section{Acknowledgements}

The calculations have been carried out at CESGA (CSIC-Xunta de Galicia), Westgrid (Canada) and TCM (Cavendish Laboratory, University of Cambridge). We are particularly grateful to several staff members for their technical support, especially to Michael Rutter (TCM, Cambridge). We are also thankful to M. A. Moreno for assistance with the early dynamics calculations. Funds from the Spanish MINECO FIS2016-77726-C3-1P, MINECO/FEDER CTQ2015-65033-P and MECD Sabbatical PRX17/00126 projects are also acknowledged. R. E. is most grateful to E. Artacho for hospitality during the sabbatical stay at Cambridge.

\section{Notes and references}

1 R. Hodyss, P. V. Johnson, J. V. Stern, J. D. Goguen and I. Kanik, Icarus, 2009, 200, 338.

2 C. R. Richey and P. A. Gerakines, Astrophys. J., 2012, 759, 74. 3 V. J. Herrero, O. Gálvez, B. Maté and R. Escribano, Phys. Chem. Chem. Phys., 2010, 12, 3164. 
4 R. M. Mastrapa, S. A. Sandford, T. L. Roush, D. P. Cruikshank and C. M. Dalle Ore, Astrophys. J., 2009, 701, 1347.

5 M. P. Bernstein, D. P. Cruikshank and S. A. Sandford, Icarus, 2006, 181, 302.

6 P. A. Gerakines, J. J. Bray, A. Davies and C. R. Richey, Astrophys. J., 2005, 620, 1140.

7 W. M. Grundy, B. Schmitt and E. Quirico, Icarus, 2002, 155, 486.

8 E. Dartois, D. Deboffle and M. Bouzit, Astron. Astrophys., 2010, 514, A49.

9 J.-L. Li, R. Car, C. Tang and N. S. Wingreen, Proc. Natl. Acad. Sci. U. S. A., 2007, 104, 2626.

10 A. J. Patel, P. Varilly, S. N. Jamadagni, H. Acharya, S. Garde and D. Chandler, Proc. Natl. Acad. Sci. U. S. A., 2011, 108, 17678.

11 M. Thomas, M. Brehm, R. Fligg, P. Vöhringer and B. Kirchner, Phys. Chem. Chem. Phys., 2013, 15, 6608.

12 S. D. Ivanov, A. Witt and D. Marx, Phys. Chem. Chem. Phys., 2013, 15, 10270.

13 J.-H. Choi and M. Cho, J. Chem. Theory Comput., 2011, 7, 4097.

14 J. Hornicek, P. Kaprálová and P. Bour, J. Chem. Phys., 2007, 127, 84502.

15 M. Praprotnik and D. Janezic, J. Chem. Phys., 2005, 122, 174103. 16 D. P. Cruikshank, W. M. Grundy, F. E. DeMeo, M. W. Buie, R. P. Binzel, D. E. Jennings, C. B. Olkin, J. W. Parker, D. C. Reuter, J. R. Spencer, S. A. Stern, L. A. Young and H. A. Weaver, Icarus, 2015, 246, 82.

17 W. M. Grundy, et al., Science, 2016, 351, aad9189.

18 Special issue R. P. Binzel, C. B. Olkin, L. A. Young and P. D. Nicholson, Icarus, 2017, 287, 1-334.

19 A. C. Adwin Boogert, P. A. Gerakines and D. C. B. Whittet, Annu. Rev. Astron. Astrophys., 2015, 53, 541-581.

20 O. Grasset, J. Castillo-Rogez, T. Guillot, L. N. Fletcher and F. Tosi, Space Sci. Rev., 2017, 212, 835.

21 K. I. Öberg, A. C. A. Boogert, K. M. Pontoppidan, G. A. Blake, N. J. Evans, F. Lahuis and E. F. van Dishoeck, Astrophys. J., 2008, 678, 1032.

22 O. Gálvez, B. Maté, V. J. Herrero and R. Escribano, Astrophys. J., 2009, 703, 2101.

23 R. M. Escribano, G. M. Muñoz-Caro, G. A. Cruz-Díaz, Y. Rodríguez-Lazcano and B. Maté, Proc. Natl. Acad. Sci. U. S. A., 2013, 110, 12899.

24 R. Escribano, E. Artacho, A. Kouchi, T. Hama, Y. Kimura, H. Hidaka and N. Watanabe, Phys. Chem. Chem. Phys., 2017, 19, 7280 .

25 P. C. Gómez and R. Escribano, Phys. Chem. Chem. Phys., 2017, 19, 26582.

26 P. D. Dopieralski, Z. Latajk and I. Olovsson, Chem. Phys. Lett., 2009, 476, 223.

27 P. Ghesquière, T. M. Mineva, D. Talbi, P. Theulé, J. A. Noble and T. Chiavassa, Phys. Chem. Chem. Phys., 2015, 17, 11455.

28 P. L. Silvestrelli, M. Bernasconi and M. Parrinello, Chem. Phys. Lett., 1997, 277, 478.
29 R. D. King-Smith and D. Vanderbilt, Phys. Rev. B: Condens. Matter Mater. Phys., 1993, 47, 1651.

30 Materials Studio (http://accelrys.com/products/materials. studio) 2014.

31 S. J. Clark, M. D. Segall, C. J. Pickard, P. J. Hasnip, M. J. Probert, K. Refson and M. C. Payne, First principles methods using CASTEP, Z. Kristallogr., 2005, 220(5-6), 567.

32 S. Grimme, J. Comput. Chem., 2006, 27, 1787.

33 E. R. McNellis, J. Meyer and K. Reuter, Phys. Rev. B: Condens. Matter Mater. Phys., 2009, 80, 205414.

34 D. Sánchez-Portal, P. Ordejón and E. Canadell, Struct. Bonding, 2004, 113, 103.

35 J. M. Soler, E. Artacho, J. D. Gale, A. García, J. Junquera, P. Ordejón and D. Sánchez-Portal, J. Phys.: Condens. Matter, 2002, 14, 2745.

36 P. Ordejón, E. Artacho and J. M. Soler, Phys. Rev. B: Condens. Matter Mater. Phys., 1996, 53, 10441.

37 F. Corsetti, M.-V. Fernández-Serra, J. M. Soler and E. Artacho, J. Phys.: Condens. Matter, 2013, 25, 435504.

38 B. Hammer, L. B. Hansen and J. K. Norskov, Phys. Rev. B: Condens. Matter Mater. Phys., 1999, 59, 7413.

39 J. Moreno and J. M. Soler, Phys. Rev. B: Condens. Matter Mater. Phys., 1992, 45, 13891.

40 D. Sánchez-Portal, I. Souza and R. M. Martin, AIP Conf. Proc., 2000, 535, 111.

41 D. Frenkel and B. Smit, Understanding Molecular Simulation, Academic Press, 2001.

42 G. Molpeceres, M. A. Satorre, J. Ortigoso, C. Millán, R. Escribano and B. Maté, Astrophys. J., 2016, 825, 156.

43 M. Bouilloud, N. Fray, Y. Benilan, H. Cottin, M.-C. Gazeau and A. Jolly, Mon. Not. R. Astron. Soc., 2015, 451, 2145.

44 J. He, K. Acharyya and G. Vidali, Astrophys. J., 2016, 823, 56. 45 I. B. Schmitt, S. Philippe, W. M. Grundy, D. C. Reuter, R. Côte, E. Quirico, S. Protopapa, L. A. Young, R. P. Binzel, J. C. Cook, D. P. Cruikshank, C. M. Dalle Ore, A. M. Earle, K. Ennico, C. J. A. Howett, D. E. Jennings, I. R. Linscott, A. W. Lunsford, C. B. Olkin, A. H. Parker, J. W. Parker, K. N. Singer, J. R. Spencer, J. A. Stansberry, S. A. Stern, C. C. C. Tsang, A. J. Verbiscer, H. A. Weaver and the New Horizons Science Team, Icarus, 2017, 287, 229.

46 S. Protopapa, W. M. Grundy, D. C. Reuter, D. P. Hamilton, C. M. Dalle Ore, J. C. Cook, D. P. Cruikshank, B. Schmitt, S. Philippe, E. Quirico, R. P. Binzel, A. M. Earle, K. Ennico, C. J. A. Howett, A. W. Lunsford, C. B. Olkin, A. Parker, K. N. Singer, A. Stern, A. J. Verbiscer, H. A. Weaver, L. A. Young and the New Horizons Science Team, Icarus, 2017, 287, 218.

47 B. Hapke, Theory of Reflectance and Emittance Spectroscopy, Topics in Remote Sensing, Cambridge University Press, Cambridge, UK, 1993.

48 R. M. Mastrapa, M. P. Bernstein, S. A. Sandford, T. L. Roush, D. P. Cruikshank and C. M. Dalle Ore, Icarus, 2008, 197, 307. 